\title{
News from the Secretariat
}

\section{Creating the sections}

In order to thoroughly examine the different problems arising from the various specific fields of Psychiatry, and to obtain and circulate information, and also to establish working relationships with the diverse national and international Organisations in the same fields and achieve a better co-ordination, AEP has constituted sections that have their own directing committee. These sections can organise their own symposia and colloquia but always under the supervision of AEP. They are autonomous regarding their scientific program and the admittance of their members, who must nevertheless be members of AEP. 